This is a peer-reviewed, accepted author manuscript of the following article: Su, W., Ho, M. T., Zhang, Y., \& Wu, L. (2020). GSIS: an efficient and accurate numerical method to obtain the apparent gas permeability of porous media. Computers and Fluids, 206, [104576]. https:// doi.org/10.1016/j.compfluid.2020.104576

\title{
GSIS: an efficient and accurate numerical method to obtain the apparent gas permeability of porous media
}

\author{
Wei Su${ }^{\mathrm{a}}$, Minh Tuan $\mathrm{Ho}^{\mathrm{a}}$, Yonghao Zhang ${ }^{\mathrm{a}}$, Lei $\mathrm{Wu}^{\mathrm{b}, *}$ \\ a James Weir Fluids Laboratory, Department of Mechanical and Aerospace Engineering, University of \\ Strathclyde, Glasgow G1 1 XJ, UK \\ ${ }^{b}$ Department of Mechanics and Aerospace Engineering, Southern University of Science and Technology, \\ Shenzhen 518055, China
}

\begin{abstract}
The apparent gas permeability (AGP) of a porous medium is an important parameter to predict the production of unconventional gas. The Klinkenberg correlation, which states that the ratio of the AGP to the intrinsic permeability is approximately a linear function of reciprocal mean gas pressure, is one of the most popular estimations to quantify AGP. However, due to the difficulty in defining the characteristic flow length in complex porous media where the rarefied gas flow is multi-scale, the slope in Klinkenberg correlation varies significantly for different geometries such that a universal expression seems impossible. In this paper, by solving the gas kinetic equation using the general synthetic iterative scheme (GSIS), we compute the AGP in porous media that are represented by Sierpinski fractals and pore body/throat systems. With the abilities of fast convergence to steady-state solution and asymptotic preserving of Navier-Stokes limit, it is shown that GSIS is a promising tool to simulate low-speed rarefied gas flow through complex multiscale geometries. A new definition of the characteristic flow length is proposed as a function of porosity, tortuosity and intrinsic permeability of porous media, which enables to find a unique slope in the Klinkenberg correlation for all the considered geometries. This research also shows that the lattice Boltzmann method using simple wall scaling for the effective shear viscosity is not able to predict the AGP of porous media.
\end{abstract}

\section{Introduction}

Unconventional gas consisting primarily of methane has become increasingly important in world energy supply due to its great potential to offset the decline in conventional gas production. Although the industry has been focusing on a rapid production, fundamental scientific questions regarding the flow behavior in ultra-tight porous media remain to be answered for economical production. In unconventional reservoirs, gas is often sourced from

\footnotetext{
*Corresponding author:

Email address: wul@sustech.edu.cn (Lei Wu) 
sedimentary rock, e.g. organic-rich shale, which is composed of fine-grained material and has pore size in the range of nanometer [1]. The flow dynamics in such unconventional reservoirs is determined by different physical processes [2]: advection and diffusion of compressed gas in pore space, adsorption and desorption in solid surface, and dissolution of soluble gas in organic material. In this paper, we focus on the transport of free gas and try to obtain an improved understanding of the apparent gas permeability (APG) that describes how fast the gas can be extracted from ultra-tight porous media. This is important, since the AGP at representative elementary volume scale is a critical parameter in upscaling equations that predict the gas production and lifespan of gas wells.

\subsection{Gas flow in conventional reservoirs}

For Stokes flow in highly permeable porous media, Darcy's law states that the total discharge $Q$ (units of volume per time) is proportional to the pressure gradient $\nabla p$ in the following form

$$
Q=-\frac{k_{\infty} A}{\mu} \nabla p
$$

where $\mu$ is the shear viscosity of fluid and $A$ is the cross-section area of a porous medium. The intrinsic permeability $k_{\infty}$, which is independent of the working fluid, reflects the geometry and topology of a porous medium only. For instance, a circular tube of diameter $D$ has $k_{\infty}=$ $D^{2} / 32$. For complex porous media, $k_{\infty}$ is not only a function of effective pore size but also other parameters, such as the effective porosity, tortuosity and constriction factor [3, 4, 5, 6].

Porosity $\epsilon$ is the fraction of connected pore volume in the total volume of a porous medium. Tortuosity $\tau$ is a measure of the length of streamlines, which is usually defined as the ratio of the shortest path $L_{\mathrm{s}}$ traversed by a fluid particle to the straight-line distance $L$ between two interconnected points in pore space [7]. A larger tortuosity indicates that fluid needs to travel longer in porous medium for a fixed $L$. Furthermore, constriction/expansion of pores, which converges/diverges the streamlines, also affects permeability. This effect is estimated by the constriction factor $C$ as follows: for a straight pore channel of length $L$, constriction factor $C$ is defined as [6]

$$
C=\frac{1}{L^{2}} \int_{0}^{L} A_{\mathrm{p}}^{2}\left(x_{1}\right) \mathrm{d} x_{1} \int_{0}^{L} \frac{1}{A_{\mathrm{p}}^{2}\left(x_{1}\right)} \mathrm{d} x_{1},
$$

where $A_{\mathrm{p}}\left(x_{1}\right)$ is the cross-sectional area of pore space at location $x_{1}$. The intrinsic permeability in two-dimensional (2D) porous media with complex structures can be related to these geometrical parameters as [5]

$$
k_{\infty}=\frac{L_{\mathrm{e}}^{2} \epsilon}{12 \tau^{2} C}
$$

where $L_{\mathrm{e}}$ is the effective pore size. Note that when $\epsilon=\tau=C=1$ we have $k_{\infty}=L_{\mathrm{e}}^{2} / 12$, which is exactly the intrinsic permeability for two parallel plates separated by a distance $L_{\mathrm{e}}$. 


\subsection{Gas flow in unconventional reservoirs}

The intrinsic permeability in unconventional reservoirs is extremely low. Nevertheless, the gas permeability measured experimentally can be significantly larger than the intrinsic permeability [8, 9], which is called AGP in order to distinguish it from the intrinsic one. Through systematic experimental investigations Klinkenberg concluded that the AGP $\left(k_{\mathrm{a}}\right)$ can be approximately expressed as [8]

$$
k_{\mathrm{a}}=k_{\infty}\left(1+\frac{b}{\bar{p}}\right)
$$

where $b$ is the correction factor and $\bar{p}$ is the mean gas pressure.

The variation of AGP with respect to the gas pressure is due to the rarefaction effect, which kicks in when the mean free path of gas molecules is comparable to or even larger than the characteristic flow length $L_{\mathrm{c}}$ in ultra-tight porous media. The degree of gas rarefaction is usually characterized by the Knudsen number $K n$

$$
K n=\frac{\mu\left(T_{0}\right)}{\bar{p} L_{\mathrm{c}}} \sqrt{\frac{\pi R T_{0}}{2}}
$$

where $\mu\left(T_{0}\right)$ is the shear viscosity of gas at a reference temperature $T_{0}$ and $R$ is the gas constant. Gas flow is usually categorized into four regimes. When $K n \lesssim 0.001$, gas flow is within the continuum regime in which the traditional Navier-Stokes equations and Darcy's law are accurate. When $0.001 \lesssim K n \lesssim 0.1$, the gas flow is in the slip regime where inadequate intermolecular collisions lead to velocity slip at solid surface, and therefore the permeability is enhanced. Note that the Navier-Stokes equations are still valid in the bulk region, i.e. a few molecular mean free paths away from the solid surface [10]. As $K n$ increases further, rarefaction effect modifies the constitutive relations in the transition $(0.1 \lesssim K n \lesssim$ $10)$ and free-molecular $(10 \lesssim K n)$ regimes such that Newton's law of viscosity and Fourier's law of heat conduction do not hold anymore [11]. As a consequence, the traditional NavierStokes equations completely lose their validity and the gas kinetic equation is enssential to describe the rarefied gas dynamics.

The rarefaction effect of gas flow through ultra-tight porous media has been extensively studied with the aim to reveal the correlation between AGP and Knudsen number (or gas pressure). Experimental works were conducted and the expression for the correction factor $b$ in the Klinkenberg correction has been obtained in terms of porous media properties, e.g. the intrinsic permeability and porosity [12, 13, 14]. Since it was obtained through data fitting, the relation varies for different datasets [15]. For practical use, a universal expression for $b$ in Eq. (4) is highly desirable. Theoretical analysis has also been carried out to find more general correlations. By applying the Navier-Stokes equations with first-order slip velocity boundary condition to the Poiseuille flow through a straight circular tube, it is found that the Klinkenberg correction (4) can be expressed as [16]

$$
k_{\mathrm{a}}=k_{\infty}(1+4 K n),
$$


while based on the second-order slip boundary condition and the observation that the asymptotic value of normalized mass flow rate for channel flow at high Knudsen number is constant, the following correlation is proposed beyond the slip flow regime [17]

$$
k_{\mathrm{a}}=k_{\infty}(1+\beta K n)\left(1+\frac{4 K n}{1+K n}\right),
$$

where $\beta=\left(128 / 15 \pi^{2}\right) \tan ^{-1}\left(4 K n^{0.4}\right)$ is an empirical fitting from the numerical solution of Boltzmann equation [16, 18].

Note that in Eqs. (6) and (7) the characteristic flow length $L_{\mathrm{c}}$ used in the definition of Knudsen number is the tube radius. For complex porous media, it is assumed that these forms of correlation still hold but the characteristic flow length (or equivalently, the Knudsen number) needs to be chosen properly. In some works, the Knudsen number is expressed in terms of the intrinsic permeability, the effective porosity and the mean gas pressure as [16]:

$$
K n=a_{0} \frac{1}{\bar{p}} k_{\infty}^{a_{1}} \epsilon^{a_{2}}
$$

where the coefficients $a_{0}, a_{1}$ and $a_{2}$ are calibrated by certain experimental data, thus are not universal. An improved relation between the effective pore size and the intrinsic permeability is proposed by treating the flow passages in porous media as a bundle of tortuous capillary tubes [18; the formulation is similar to Eq. (3) but without consideration of the constriction factor. More complex formula for $K n$ in terms of the intrinsic permeability and geometrical features of the porous media can be found in Ref. [19], which is derived based on the fractal model. Recently, Yang and Weigand used the effective pore size $L_{\mathrm{e}}$ in Eq. (3) as the characteristic flow length and found that the enhancement in gas permeability in different porous structures can be universally described by the Knudsen number defined using this effective pore size [20]. However, in their numerical simulations, the impermeable grains are of equal size. Whether the universal definition of $K n$ works for other porous media remains to be assessed.

\subsection{Numerical methods to solve rarefied gas flow in porous media}

With the technological advance in high-performance computing, rarefied gas flow through complicated geometry can now be simulated based on the gas kinetic theory that is indispensable for the description of gas flow in all flow regimes. The direct simulation Monte Carlo (DSMC) method was used to investigate the pressure-driven gas flows through porous media [21, 22, 23, 20]. However, due to its statistical noise and the limitation that the spatial cell size should be smaller than the mean free path of gas molecules, only a few data points on the AGP were obtained, which may hinder the discovery of universal correlation.

Alternatively, the gas kinetic equation can be solved by the discrete velocity method (DVM), which is promising at producing statistical-noise-free solution for low-speed gas flow that is typical in porous media [24, 25, 26]. In the transition and free-molecular flow regimes, the conventional iterative scheme (CIS) can find the steady-state solution of gas kinetic equation efficiently and accurately. However, CIS converges extremely slowly for 
near-continuum flow [27]. Worse still, its results are contaminated by numerical dissipation if the spatial cell size is larger than the molecular mean free path. This poses a severe problem to simulate multi-scale rarefied gas flows in complex porous media, since in some region the gas flow is highly rarefied while in other parts the gas flow falls in the nearcontinuum regime. To remove these deficiencies, the general synthetic iterative scheme (GSIS) 28] is recently developed to find steady-state solution of general rarefied gas flow within dozens of iterations at any Knudsen number. What's more, the GSIS asymptotically preserves the Navier-Stokes limit, so that the spatial cell size can be much larger than the molecular mean free path. These advantages make the GSIS a promising tool to tackle the difficulty in simulating multi-scale rarefied gas flows.

It should be noted that recently, the lattice Boltzmann method (LBM) has been used to simulate the rarefied gas flows through porous media [29, 30, 31, 32, 33, 34]. Rooted from the gas kinetic theory, LBM is a powerful tool originally designed to solve the incompressible Navier-Stokes equations based on the Bhatnager-Gross-Krook (BGK) equation [35] and the bounce-back scheme (in which gas molecules colliding with the wall simply reverse the direction of velocity [36]). Since one of the signatures of rarefied gas flow is the velocity slippage at the wall, the bounce-back and specular-reflection boundary conditions are combined to simulate the slip motion of gas at solid wall [37, 38]. To improve LBM for more rarefied gas flow, Shan et al. proposed to use high-order Gauss-Hermite quadrature to approximate the velocity distribution function [39], while Zhang et al. introduced the wall-scaling relaxation time to the traditional LBM to describe the effective viscosity within the Knudsen layer [40]. Further development was made by Guo et al., where in addition to the wall scaling relaxation time, a generalized model of combined bounce-back and specular-reflection was proposed to realize the second-order slip boundary condition [41]; their multiple relaxation time (MRT) LBM is able to prediction the velocity profile and mass flow rate of Poiseuille flow between two parallel plates up to the free-molecular flow regime. However, by comparing with the accurate solution of the BGK model equation, it was found that the accuracy of MRT-LBM is reduced significantly in simulating rarefied gas flow through complex geometry, such as rough surface and porous media [42.

The aims of this paper are three-fold. First, we will test the efficiency and accuracy of the newly introduced GSIS in simulating multi-scale rarefied gas flow through porous media. Second, based on the accurate solution of gas kinetic equation, we will further assess the accuracy of LBM that was recently applied to find the AGP through porous media represented by the Sierpinski fractal model [29] and a simplified pore body/throat system [30]. Finally, we will find a unique expression of the slope coefficient in the Klinkenberg correction that quantifies AGP in the slip flow regime.

The remainder of this paper is organized as follows. The considered geometries are described in Sec. 2, In Sec. 3, the linearized BGK equation and GSIS are introduced. Numerical results and discussions are presented in Sec. 4, including the comparison between CIS and GSIS in terms of efficiency and accuracy, comparison between GSIS and LBM for the calculated intrinsic permeabilities, flow fields and AGP through various porous media. A universal expression for the Klinkenberg correction for the two types of geometry is proposed in Sec. 4. Finally, conclusions are given in Sec. 5 . 

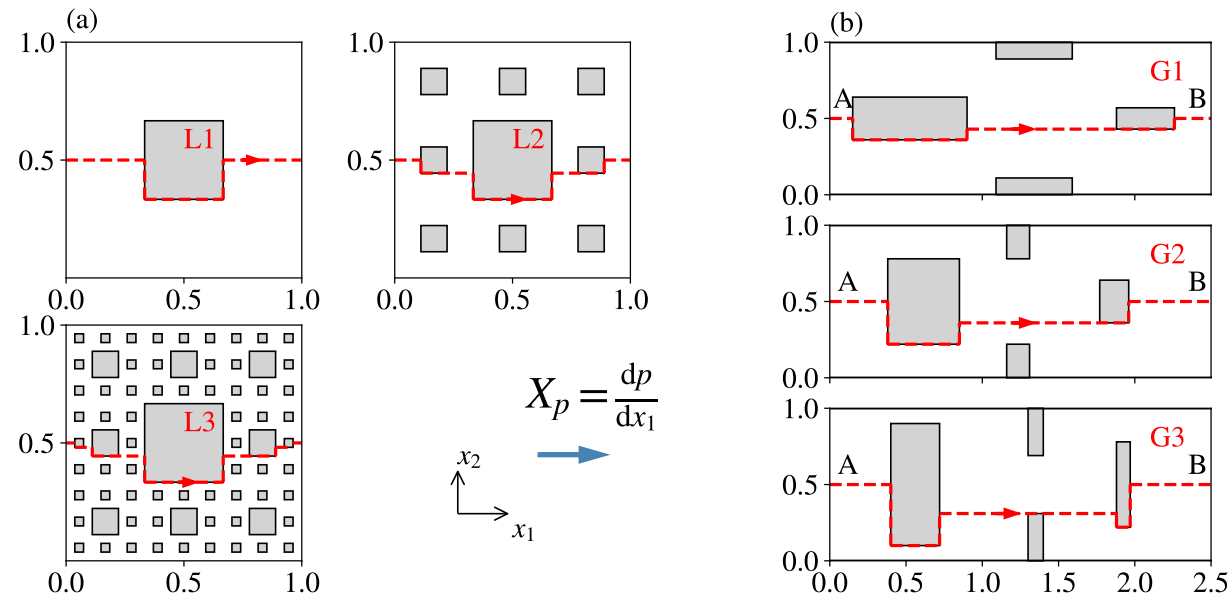

Figure 1: Schematic geometries of the porous media, where the white and shaded regions represent the pore voids and impermeable obstacles, respectively. (a) 'L1', 'L2' and 'L3' denote the first three levels of Sierpinski fractals. (b) Pore body/throat system: 'G1', 'G2' and 'G3' denote three geometries with fixed porosity but varying tortuosity. The red dash line in each subfigure shows the imaginary flow path.

\section{Statement of the problem}

In this work, we consider 2D porous media in Fig. 1, where the white and shaded regions represent the pore voids and impermeable obstacles, respectively. The first set of geometries is generated recursively by the Sierpinski fractal model [43, 44]. That is, a square of size 1 by 1 is cut into 9 congruent sub-squares in a $3 \times 3$ grid, and the central sub-square is modeled as an obstacle. The same procedure is then applied to the remaining 8 sub-squares for the next level of recursion. Resulting geometries generated by the first three levels of recursion are shown in Fig. 1(a), which are marked as 'L1', 'L2' and 'L3', respectively. The second set of geometries is shown in Fig. 1(b). The simple pore body/throat system has four rectangles that are used to represent impermeable obstacles. Altering the sizes and positions of these rectangles changes the porosities and tortuosities. In order to investigate the influence of tortuosity on gas permeability, we consider three different geometries (marked as 'G1', 'G2' and 'G3'), which have the same porosity of about 0.85 , but different values of tortuosity. Note that the definition of tortuosity is not unique [3]; here it is defined as the ratio of the shortest path that a fluid particle traverses and the straight-line distance between its original source and destination. As shown in Fig. 1(b), we consider an imaginary flow path, the ends of which (labeled as 'A' and 'B') are at the centers of the inlet and outlet, respectively. For simplification, we assume that the fluid only makes quarter turn when they encounter the obstacles. Thus, the geometric tortuosities for G1, G2 and G3 are about 1.11, 1.22 and 1.39, respectively. The same procedure can be applied to the Sierpinski fractals, and it is found that L1, L2 and L3 have the same tortuosity of 1.33.

The geometrical properties for the six porous media are summarized in Table 1. In addition to their porosity $\epsilon$ and tortuosity $\tau$, the constriction factor $C$ defined in Eq. (2) are also listed. We will calculate the AGP of these porous media and check whether it is possible to obtain a unique expression for the slope factor. To this end, we assume that 
Table 1: Porosity $\epsilon$, tortuosity $\tau$ and constriction factor $C$ for the six geometries shown in Fig. 1 .

\begin{tabular}{cccccccc}
\hline & \multicolumn{3}{c}{ Sierpinski fractals } & & \multicolumn{3}{c}{ pore body/throat system } \\
& L1 & L2 & L3 & & G1 & G2 & G3 \\
\cline { 2 - 4 }$\epsilon$ & 0.8889 & 0.7901 & 0.7023 & & 0.8507 & 0.8470 & 0.8526 \\
$\tau$ & 1.3333 & 1.3333 & 1.3333 & & 1.1120 & 1.2240 & 1.3920 \\
$C$ & 1.1621 & 1.3358 & 1.5396 & & 1.0784 & 1.5314 & 3.6375 \\
\hline
\end{tabular}

gas flows through the porous media from left to right in the $x_{1}$ direction, driven by a small enough constant pressure gradient $X_{\mathrm{p}}$. We are interested in the velocity field and AGP over a range of Knudsen number.

\section{Gas kinetic theory and numerical methods}

\subsection{The linearized BGK equation}

In gas kinetic theory, gas system state is described by the velocity distribution function, which is a function of the spatial position $\boldsymbol{x}$, molecular velocity $\boldsymbol{v}$, and time $t$. Evolution of the velocity distribution function $f$ is governed by the following Boltzmann equation

$$
\frac{\partial f}{\partial t}+\boldsymbol{v} \cdot \frac{\partial f}{\partial \boldsymbol{x}}+\boldsymbol{a} \cdot \frac{\partial f}{\partial \boldsymbol{x}}=\mathcal{C}(f)
$$

where $\boldsymbol{x}=\left(x_{1}, x_{2}, x_{3}\right)$ is the spatial coordinates normalized by the height of a porous medium $H, \boldsymbol{v}=\left(v_{1}, v_{2}, v_{3}\right)$ is the three-dimensional molecular velocity space normalized by the most probable speed $v_{\mathrm{m}}=\sqrt{2 R T_{0}}, t$ is the time normalized by $H / v_{\mathrm{m}}$, the velocity distribution function $f$ is normalized by $\rho_{0} v_{\mathrm{m}}^{3} / M$ with $M$ being the molecular mass, $\boldsymbol{a}=\left(a_{1}, a_{2}, a_{3}\right)$ is the external acceleration normalized by $v_{\mathrm{m}}^{2} / H$. Finally, $\mathcal{C}$ is the Boltzmann collision operator, which is usually replaced by the BGK model [35.

For gas flow through porous media, the pressure gradient $X_{\mathrm{p}}=\mathrm{d} p / \mathrm{d} x_{1}$, which is normalized by $\bar{p} / H$, is small. Then, we can linearize the velocity distribution function about the global equilibrium $f_{\text {eq }}$ by introducing a small perturbation $h$

$$
f=f_{\text {eq }}(1+h), \quad f_{\text {eq }}=\frac{\exp \left(-|\boldsymbol{v}|^{2}\right)}{\pi^{3 / 2}},
$$

where $h$ is governed by the following linearized BGK equation [45]

$$
\begin{array}{r}
\frac{\sqrt{\pi}}{2 K n} h+\boldsymbol{v} \cdot \frac{\partial h}{\partial \boldsymbol{x}}=-X_{\mathrm{p}} v_{1}+\frac{\sqrt{\pi}}{2 K n} \mathcal{L}(\varrho, \boldsymbol{u}, \tau), \\
\mathcal{L}(\varrho, \boldsymbol{u}, \tau)=\varrho+2 \boldsymbol{u} \cdot \boldsymbol{v}+\tau\left(|\boldsymbol{v}|^{2}-\frac{3}{2}\right),
\end{array}
$$

with $\varrho$ being the perturbed flow density, $\boldsymbol{u}=\left(u_{1}, u_{2}\right)$ the bulk flow velocity and $\tau$ the perturbed flow temperature. Here, we have omitted the derivative with respect to the time since we are only interested in the steady-state solution. 


\subsection{Discrete velocity method for 2D problem}

The perturbation $h$ in Eq. (11) is defined in a six-dimensional phase space. For 2D problem, however, the phase space can be cast into a four-dimensional one by introducing the following two reduced velocity distributions:

$$
\begin{array}{r}
\Phi\left(\boldsymbol{x}_{2 \mathrm{D}}, \boldsymbol{v}_{2 \mathrm{D}}\right)=\frac{1}{\sqrt{\pi}} \int \exp \left(-v_{3}^{2}\right) h \mathrm{~d} v_{3}, \\
\Psi\left(\boldsymbol{x}_{2 \mathrm{D}}, \boldsymbol{v}_{2 \mathrm{D}}\right)=\frac{1}{\sqrt{\pi}} \int\left(v_{3}^{2}-\frac{1}{2}\right) \exp \left(-v_{3}^{2}\right) h \mathrm{~d} v_{3} .
\end{array}
$$

Note that we use $\boldsymbol{x}_{2 \mathrm{D}}=\left(x_{1}, x_{2}\right)$ and $\boldsymbol{v}_{2 \mathrm{D}}=\left(v_{1}, v_{2}\right)$ to denote the 2D physical space and molecular velocity space, respectively. The governing equations for $\Phi$ and $\Psi$ are obtained by multiplying Eq. (11) with $\exp \left(-v_{3}^{2}\right) / \sqrt{\pi}$ and $\left(v_{3}^{2}-1 / 2\right) \exp \left(-v_{3}^{2}\right) / \sqrt{\pi}$, respectively, and integrating the resulting equations with respect to $v_{3}$ :

$$
\begin{array}{r}
\frac{\sqrt{\pi}}{2 K n} \Phi+\boldsymbol{v}_{2 \mathrm{D}} \cdot \frac{\partial \Phi}{\partial \boldsymbol{x}_{2 \mathrm{D}}}=-X_{\mathrm{p}} v_{1}+\frac{\sqrt{\pi}}{2 K n} \mathcal{L}_{\Phi}, \\
\frac{\sqrt{\pi}}{2 K n} \Psi+\boldsymbol{v}_{2 \mathrm{D}} \cdot \frac{\partial \Psi}{\partial \boldsymbol{x}_{2 \mathrm{D}}}=\frac{\sqrt{\pi}}{2 K n} \mathcal{L}_{\Psi}
\end{array}
$$

where $\mathcal{L}_{\Phi}(\varrho, \boldsymbol{u}, \tau)=\varrho+2 \boldsymbol{u} \cdot \boldsymbol{v}_{2 \mathrm{D}}+\tau\left(\left|\boldsymbol{v}_{2 \mathrm{D}}\right|^{2}-1\right)$ and $\mathcal{L}_{\Psi}(\tau)=\tau / 2$. The perturbed density $\varrho$, bulk velocity $\boldsymbol{u}$ and perturbed temperature are obtained from the velocity moment of the perturbed velocity distribution function as

$$
\begin{array}{r}
\varrho=\int \Phi f_{\mathrm{eq}}^{2 \mathrm{D}} \mathrm{d} \boldsymbol{v}_{2 \mathrm{D}}, \\
\boldsymbol{u}=\int \boldsymbol{v} \Phi f_{\mathrm{eq}}^{2 \mathrm{D}} \mathrm{d} \boldsymbol{v}_{2 \mathrm{D}}, \\
\tau=\frac{2}{3} \int\left[\left(\left|\boldsymbol{v}_{2 \mathrm{D}}\right|^{2}-1\right) \Phi+\Psi\right] f_{\mathrm{eq}}^{2 \mathrm{D}} \mathrm{d} \boldsymbol{v}_{2 \mathrm{D}},
\end{array}
$$

where $f_{\text {eq }}^{2 D}=\exp \left(-\left|\boldsymbol{v}_{2 \mathrm{D}}\right|^{2}\right) / \pi$. For conciseness, we will omit the subscript/superscript '2D' in the remainder of the paper.

To numerically solve Eq. (13), the discrete velocity method (DVM) is employed, in which the continuous molecular velocity space is first represented by a set of discrete velocities. Theoretically, any set of quadrature points for the approximation of integration with respect to $\boldsymbol{v}$ is applicable. However, for an accurate approximation, especially for efficient capture of the discontinuity and rapid variation in velocity distribution function at large Knudsen number, the following non-uniform discretization is more preferable:

$$
v_{i}^{j_{i}}=\frac{j_{i}^{3}}{\left(N_{\mathrm{v}}-1\right)^{3}} v_{\max }, \quad i=1 \text { or } 2,
$$

where the index $j_{i}=-N_{\mathrm{v}}+1,-N_{\mathrm{v}}+3, \ldots, N_{\mathrm{v}}-1$ with $N_{\mathrm{v}}$ being the number of discrete velocities. Note that the molecular velocity space can also be discretized by the Gauss-Hermite 
quadrature as that used in LBM, however this type of discretization is not accurate/efficient for flow at large Knudsen number [42].

It should be mentioned that the MRT-LBM using very limited number of discrete velocities, e.g. the D2Q9 scheme for 2D problem, is able to predict the Poiseuille flow between two parallel plates with good accuracy up to the free-molecular flow regime. This is achieved by introducing gas kinetic boundary condition, as well as effective geometry-dependent viscosity that is a function of the gas-wall distance. However, this scheme is only designed for planar walls [41]. In Sec. 4 we will show that the MRT-LBM loses its accuracy for complex geometry such as porous medium.

\subsection{Conventional iterative scheme}

After discretization in the phase space, the steady-state solution of Eq. (13) is usually solved straightforwardly by the following conventional iterative scheme (CIS)

$$
\begin{array}{r}
\frac{\sqrt{\pi}}{2 K n} \Phi^{j, k+1}+\boldsymbol{v}^{j} \cdot \frac{\partial \Phi^{j, k+1}}{\partial \boldsymbol{x}}=-X_{\mathrm{p}} v_{1}^{j_{1}}+\frac{\sqrt{\pi}}{2 K n} \mathcal{L}_{\Phi}^{k}, \\
\frac{\sqrt{\pi}}{2 K n} \Psi^{j, k+1}+\boldsymbol{v}^{j} \cdot \frac{\partial \Psi^{j, k+1}}{\partial \boldsymbol{x}}=\frac{\sqrt{\pi}}{2 K n} \mathcal{L}_{\Psi}^{k},
\end{array}
$$

where $\Phi^{j}\left(x_{1}, x_{2}\right)=\Phi\left(x_{1}, x_{2}, v_{1}^{j_{1}}, v_{2}^{j_{2}}\right)$ and $\Psi^{j}\left(x_{1}, x_{2}\right)=\Psi\left(x_{1}, x_{2}, v_{1}^{j_{1}}, v_{2}^{j_{2}}\right)$ represent the perturbations at discrete velocity nodes $\boldsymbol{v}^{j}=\left(v_{1}^{j_{1}}, v_{2}^{j_{2}}\right)$, while $\mathcal{L}_{\Phi}^{k}=\mathcal{L}_{\Phi}\left(\varrho^{k}, \boldsymbol{u}^{k}, \tau^{k}\right)$ and $\mathcal{L}_{\Psi}^{k}=$ $\mathcal{L}_{\Psi}\left(\tau^{k}\right)$. The superscripts $k$ and $k+1$ denote two consecutive iteration steps. The macroscopic quantities $\varrho^{k}, \boldsymbol{u}^{k}$ and $\tau^{k}$ are evaluated from Eq. (14) with the integration approximated by numerical quadrature.

During each iteration, Eq. (16) should be supplied with the gas kinetic boundary condition to determine $\Phi^{j}$ and $\Psi^{j}$ at boundary. In this paper, the left and right boundaries where gas flows in and out the porous media are modeled as periodic boundary, written as

$$
\begin{aligned}
& \Phi^{j}\left(x_{1}=0, x_{2}\right)=\Phi^{j}\left(x_{1}=\frac{L}{H}, x_{2}\right), \\
& \Psi^{j}\left(x_{1}=0, x_{2}\right)=\Psi^{j}\left(x_{1}=\frac{L}{H}, x_{2}\right),
\end{aligned}
$$

where $L$ is the length of the computational domain. The lower and upper boundaries, as well as the surfaces of the impermeable obstacles inside porous media are modeled as fully diffuse boundary. Suppose the static surfaces are at the reference temperature $T_{0}$ and the mass flux across surface is equal to zero, then the perturbations for the reflected molecules (i.e., when $\boldsymbol{v}^{j} \cdot \boldsymbol{n}>0, \boldsymbol{n}$ is the outward unit normal vector at the solid surface) are given by

$$
\begin{aligned}
& \Phi^{j}=-\frac{2}{\sqrt{\pi}} \sum_{\boldsymbol{v}^{j} \cdot \boldsymbol{n}<0} \boldsymbol{v}^{j} \cdot \boldsymbol{n} \Phi^{j} \exp \left(-\left|\boldsymbol{v}^{j}\right|^{2}\right) \Delta \boldsymbol{v}^{j}, \\
& \Psi^{j}=0,
\end{aligned}
$$

where $\Delta \boldsymbol{v}^{j}=\Delta v_{1}^{j_{1}} \Delta v_{2}^{j_{2}}$ is the velocity intervals with $\Delta v_{i}^{j_{i}}=3 v_{\max } j_{i}^{2} /\left(N_{\mathrm{v}}-1\right)^{3}$, since the non-uniform velocity grid (15) is used. 


\subsection{General synthetic iterative scheme}

The CIS is efficient and accurate to find steady-state solution in the transition and freemolecular flow regimes. However, it converges extremely slowly for near-continuum flow, where the "converged" solution is usually wrong due to large numerical dissipation [27, 46]. To facilitate fast convergence and reduce the numerical viscosity in the near-continuum flow regime, the GSIS is recently proposed [28]. The key ingredient of GSIS is that a set of macroscopic equations is simultaneously solved with the kinetic equation (16), from which the macroscopic flow properties are obtained to guide the evolution of molecular velocity distribution function. Due to the fact that the constitutive relations involving in the synthetic macroscopic equations explicitly contain not only the Newton's law of viscosity and Fourier's law of thermal conduction but also high-order terms exactly derived from the kinetic equation, GSIS has the capacities to achieve fast convergence, asymptotically preserve the Navier-Stokes limit, and keep accuracy for rarefied effect. For this problem, the synthetic macroscopic equations are written as

$$
\begin{array}{r}
\frac{\partial u_{1}}{\partial x_{1}}+\frac{\partial u_{2}}{\partial x_{2}}=0 \\
\frac{\partial \varrho}{\partial x_{1}}+\frac{\partial \tau}{\partial x_{1}}+\frac{\partial \sigma_{11}}{\partial x_{1}}+\frac{\partial \sigma_{12}}{\partial x_{2}}=-X_{\mathrm{p}} \\
\frac{\partial \varrho}{\partial x_{2}}+\frac{\partial \tau}{\partial x_{2}}+\frac{\partial \sigma_{12}}{\partial x_{1}}+\frac{\partial \sigma_{22}}{\partial x_{2}}=0 \\
\frac{\partial q_{1}}{\partial x_{1}}+\frac{\partial q_{2}}{\partial x_{2}}=0
\end{array}
$$

and the constitutive relations for the shear stress $\sigma_{l m}$ and heat flux $q_{l}(l, m=1$, or 2$)$ are

$$
\begin{aligned}
& \frac{\sqrt{\pi}}{2 K n} \sigma_{l m}+\frac{\partial u_{l}}{\partial x_{m}}+\frac{\partial u_{m}}{\partial x_{l}}-\frac{2}{3}\left(\frac{\partial u_{1}}{\partial x_{1}}+\frac{\partial u_{2}}{\partial x_{2}}\right) \delta_{l m}+\mathrm{HoT}_{\sigma_{l m}}=0, \\
& \frac{\sqrt{\pi}}{2 K n} q_{l}+\frac{5}{4} \frac{\partial \tau}{\partial x_{l}}+\operatorname{HoT}_{q_{l}}=0,
\end{aligned}
$$

where $\delta_{l m}$ is the Kronecker delta function. The high-order terms $\mathrm{HoT}_{\sigma_{l m}}$ and $\mathrm{HoT}_{q_{l}}$ that take account of rarefaction effects are

$$
\begin{array}{r}
\mathrm{HoT}_{\sigma_{l m}}=\sum_{n=1,2} \frac{\partial}{\partial x_{n}} \int 2 v_{n}\left[\left(v_{l} v_{m}-\frac{\delta_{l m}}{3}|\boldsymbol{v}|^{2}-\frac{\delta_{l m}}{6}\right) \Phi-\frac{\delta_{l m}}{3} \Psi\right] f_{\mathrm{eq}} \mathrm{d} \boldsymbol{v} \\
-\left[\frac{\partial u_{l}}{\partial x_{m}}+\frac{\partial u_{m}}{\partial x_{l}}-\frac{2}{3}\left(\frac{\partial u_{1}}{\partial x_{1}}+\frac{\partial u_{2}}{\partial x_{2}}\right) \delta_{l m}\right], \\
\operatorname{HoT}_{q_{l}}=\sum_{n=1,2} \frac{\partial}{\partial x_{n}} \int v_{n} v_{l}\left[\left(|\boldsymbol{v}|^{2}-2\right) \Phi+\Psi\right] f_{\mathrm{eq}} \mathrm{d} \boldsymbol{v}-\frac{5}{4} \frac{\partial \tau}{\partial x_{l}}
\end{array}
$$

The periodic boundary condition for $\varrho, \boldsymbol{u}$ and $\tau$ at the left and right boundaries is used to solve the synthetic macroscopic equations (19), while the boundary condition for the 
macroscopic flow properties at the solid surfaces is directly evaluated according to Eq. (14), in order to ensure the correct slip velocity and temperature jump.

Finally, the procedure to execute the GSIS is described as follows (where the index $j$ for the discrete velocities has been omitted):

- Step 1. When the velocity distribution functions $\Phi^{k}$ and $\Psi^{k}$ are known at the $k$ th iteration, we calculate the macroscopic flow properties $\varrho^{k}, \boldsymbol{u}^{k}$ and $\tau^{k}$ according to Eq. (14). We also calculate the velocity distribution functions $\Phi^{k+1 / 2}$ and $\Psi^{k+1 / 2}$ according to the conventional iterative scheme, i.e. we solve

$$
\begin{array}{r}
\frac{\sqrt{\pi}}{2 K n} \Phi^{k+1 / 2}+\boldsymbol{v} \cdot \frac{\partial \Phi^{k+1 / 2}}{\partial \boldsymbol{x}}=-X_{p} v_{1}+\frac{\sqrt{\pi}}{2 K n} \mathcal{L}_{\Phi}^{k}, \\
\frac{\sqrt{\pi}}{2 K n} \Psi^{k+1 / 2}+\boldsymbol{v} \cdot \frac{\partial \Psi^{k+1 / 2}}{\partial \boldsymbol{x}}=\frac{\sqrt{\pi}}{2 K n} \mathcal{L}_{\Psi}^{k}
\end{array}
$$

- Step 2. From $\Phi^{k+1 / 2}$ and $\Psi^{k+1 / 2}$, we calculate the high-order terms $\operatorname{HoT}_{\sigma_{l m}}$ and $\mathrm{HoT}_{q_{l}}$ defined in Eq. (21), as well as the macroscopic quantities $\varrho^{k+1 / 2}, \boldsymbol{u}^{k+1 / 2}$ and $\tau^{k+1 / 2}$.

- Step 3. We obtain the flow properties $\varrho^{k+1}, \boldsymbol{u}^{k+1}$ and $\tau^{k+1}$ by solving the synthetic equations (19). Note that the Newton's law of viscosity and Fourier's law of thermal conduction in the constitutive relations $(20)$ are calculated at the $(k+1)$-th iteration step, while high-order terms $\mathrm{HoT}_{\sigma_{l m}}$ and $\mathrm{HoT}_{q_{l}}$ are computed at the $(k+1 / 2)$-th iteration step.

- Step 4. The velocity distribution functions are updated to incorporate the change of macroscopic quantities

$$
\Phi^{k+1}=\Phi^{k+1 / 2}+\lambda_{\varrho}+2 \boldsymbol{v} \cdot \boldsymbol{\lambda}_{u}+\lambda_{\tau}\left(|\boldsymbol{v}|^{2}-1\right), \quad \Psi^{k+1}=\Psi^{k+1 / 2}+\frac{1}{2} \lambda_{\tau},
$$

where

$$
\begin{array}{r}
\lambda_{\varrho}=\alpha\left(\varrho^{k+1}-\varrho^{k+1 / 2}\right), \\
\lambda_{\boldsymbol{u}}=\alpha\left(\boldsymbol{u}^{k+1}-\boldsymbol{u}^{k+1 / 2}\right), \\
\lambda_{\tau}=\alpha\left(\tau^{k+1}-\tau^{k+1 / 2}\right),
\end{array}
$$

and

$$
\alpha=\frac{\min \left(K n_{\mathrm{loc}}, 1\right)}{K n_{\mathrm{loc}}},
$$

with $K n_{\text {loc }}$ being the local Knudsen number is a stabilization factor, which is introduced to make the GSIS stable when $K n_{\text {loc }}$ is large. The definition of $K n_{\text {loc }}$ used in this paper is specified in the next section.

- Step 5. The above steps are repeated until convergence. 


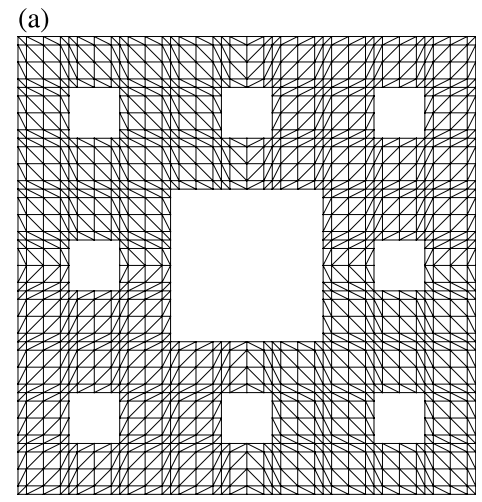

(b)

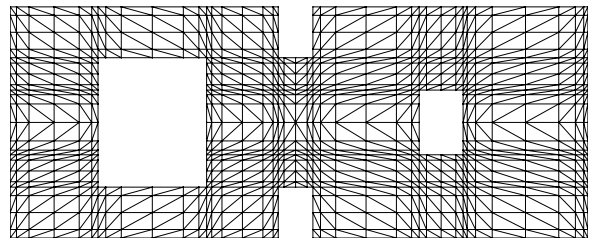

Figure 2: Schematic triangular meshes for (a) Sierpinski Carpet L2 and (b) pore body/throat system G2.

It can be proven theoretically [47] that (i) the GSIS finds the steady-state solution quickly and (ii) like the implicit unified gas kinetic scheme [48] the GSIS asymptotically preserves the Navier-Stokes limit when the Knudsen number is small. For the property (i), the fast convergence of GSIS is reached not only due to the exact inclusion of the NavierStokes constitutive relations in (20), but also in each iteration for the kinetic equation, the macroscopic synthetic equations are solved to the steady-state. For the property (ii), it is interesting to note that the coupling of streaming and collision in the evaluation of flux at the cell interface [48] is not the only way to realize the asymptotic Navier-Stokes preserving. Therefore, the kinetic equation is not necessary solved under the framework of unified gas kinetic scheme.

\section{Results and discussions}

In this paper, the 4th-order discontinuous Galerkin (DG) method is applied to discretize the governing equations (13) and (19) on triangular meshes in the physical space. The sweeping technique is employed to solve the kinetic equations to avoid solving large linear system. The hybridizable discontinuous Galerkin method [49] is applied to solve macroscopic equations. More details about the implementation of DG discretization can be found in Refs. [28, 50, 51]. Figure 2 shows the schematic triangular meshes, where refinement is placed near the solid boundaries.

In addition to the velocity field, we are also interested in the mean volumetric flow rate $\mathcal{M}$ and the AGP $\kappa_{\mathrm{a}}$. The two quantities are evaluated as

$$
\mathcal{M}=\frac{H}{L X_{\mathrm{p}}} \iint u_{1} \mathrm{~d} x_{1} \mathrm{~d} x_{2}, \quad \kappa_{a}=-\frac{2 K n}{\sqrt{\pi}} \mathcal{M} .
$$

The CIS and GSIS are used to find the steady-state solutions, where the iteration is terminated when the residual in flow velocity between two consecutive iteration steps

$$
\mathcal{R}=\frac{\iint\left(\left|\boldsymbol{u}^{k+1}\right|-\left|\boldsymbol{u}^{k}\right|\right)^{2} \mathrm{~d} x_{1} \mathrm{~d} x_{2}}{\iint\left(\left|\boldsymbol{u}^{k+1}\right|\right)^{2} \mathrm{~d} x_{1} \mathrm{~d} x_{2}}
$$



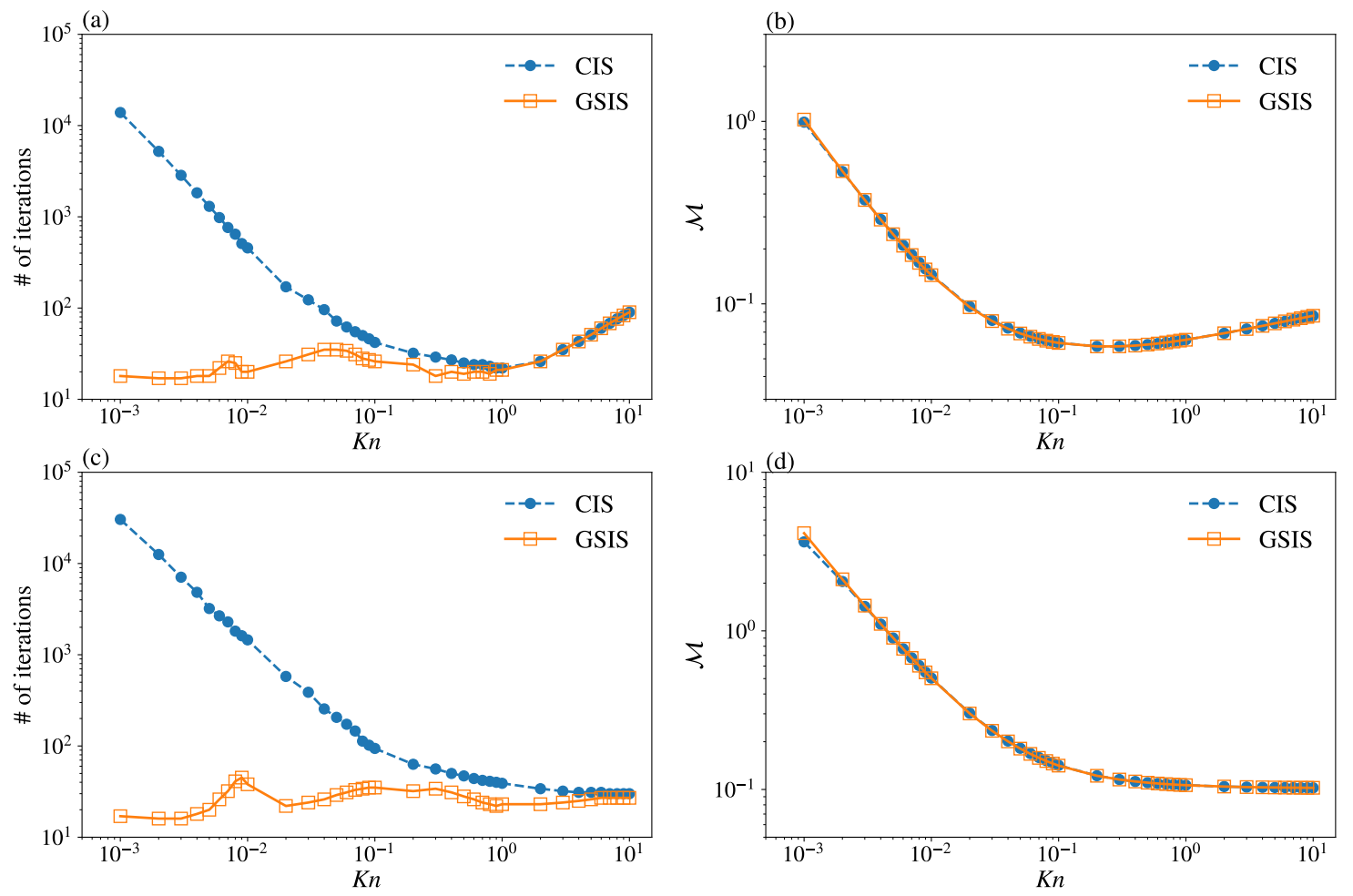

Figure 3: Comparison between CIS and GSIS in terms of the number of iterations to reach convergence $\left(\mathcal{R}<10^{-5}\right)$ and the obtained volumetric flow rate. (a, b) The second level Sierpinski fractal L2. (c, d) The pore body/throat system G2.

is less than $10^{-5}$. The local Knudsen number to calculate the stabilization factor for GSIS in Eq. (24) is estimated as $K n_{\mathrm{loc}}=K n H / H_{\min }$ with $H_{\min }$ the minimum height of the local mesh triangle.

In order to ensure the simulation results are accurate, the convergence study is performed by using different numbers of $N_{\mathrm{v}}$ and different triangular meshes. Eventually, 1024, 4608 and 16384 triangles are used for the first three levels of Sierpinski fractals, respectively, while 4088, 3488, and 3520 triangles are used for the pore body/throat system G1, G2 and G3, respectively. For the discretization in the molecular velocity space, the non-uniform discretization of $v_{\max }=4$ and $N_{\mathrm{v}}=48$ is employed for all flows with $K n \in[0.001,10]$, see Eq. (15), and the 8-point Gauss-Hermite quadrature is used to find the intrinsic permeability of each geometry at $K n=10^{-5}$. Further increment of the number of velocity points and triangles would improve the results of $\mathcal{M}$ by no more than $1 \%$.

\subsection{Efficiency and accuracy of CIS and GSIS}

Rarefied gas flows through the Sierpinski fractal L2 and the pore body/throat system G2 are solved by CIS and GSIS, when $K n \in[0.001,10]$. Figure 3(a) and (c) plot the number of iterations needed to reach the convergence criterion of $\mathcal{R}<10^{-5}$. For the flow through geometry L2 with $K n \leq 1$, the number of iterations in CIS dramatically increases as the Knudsen number decreases, while it slightly increases as $K n$ varies from 1 to 10 . 
For instance, CIS takes 13908, 22 and 90 iterations to find the steady-state solutions when $K n=0.001,1$ and 10, respectively. For the flow through G2, the number of iterations of CIS increases monotonically as $K n$ decreases: CIS takes 30393, 39 and 30 iterations to find the steady-state solutions at $K n=0.001,1$ and 10, respectively. In contrast, GSIS obtains steady-state solution within dozens of iterations at any Knudsen number. At $K n=0.001$, GSIS only needs 18 and 17 steps to reach convergence for flows through L2 and G2 geometries, respectively. Due to the fact that the CPU time consumed to solve macroscopic equations is much smaller than that for the kinetic equation, the save of CPU time for GSIS is equivalent to the save in iteration number; and this is significant when Knudsen number is small. For example, CIS costs about 280 hours to obtain the converged result for L2 geometry at $K n=0.001$ on single Intel Xeon-E5-2680 processor, while GSIS only needs 0.4 hour. Figure $3(\mathrm{~b})$ and (d) show the volumetric flow rate $\mathcal{M}$ obtained from CIS and GSIS for L2 and G2 geometries, respectively. Both schemes predict almost the same solutions in the whole Knudsen range, which proves the accuracy of GSIS.

\subsection{Comparison between $L B M$ and GSIS}

Recently, the MRT-LBM with modified viscosity and boundary condition was used to find the AGP of the Sierpinski fractals and the pore body/throat system, where the diffuse reflection condition or the combined bounce-back and specular reflection condition is applied at solid surfaces [30, 29]. However, the method may lose accuracy in simulating rarefied gas flow through complex geometry, since the limited number of discrete velocity points used in MRT-LBM are not adequate to describe the velocity distribution function [42]. Here we compare the velocity fields and the AGP obtained from LBM and GSIS.

Figure 4 plots the horizontal velocity contours and streamlines obtained from GSIS for the Sierpinski fractals at $K n=0.01,0.1$ and 1 . At each Knudsen number, as more impermeable solids are added, the porosity decreases, the flow passage becomes narrow, and the magnitude of velocity decreases. Due to the constricting and expanding of pore space, noticeable vortexes appear in the local flow field in L2 and L3 geometries. However, no vortex appears in the first level fractal for all the Knudsen numbers considered. On the contrary, LBM predicts large vortexes in the flow field of L1 geometry, see Figure 10 in Ref. [29]. Figure 5 shows the horizontal velocity contours and streamlines obtained by the GSIS for the pore body/throat systems at $K n=0.01,0.1$ and 1 . At each Knudsen number, the magnitude of horizontal velocity drops as the tortuosity increases. For each geometry, the magnitude of horizontal velocity drops as the Knudsen number becomes large. For all the considered geometries and Knudsen numbers, the largest velocity occurs in the narrowest flow passage, i.e. these beneath and above the left solid obstacle.

AGPs obtained from GSIS and MRT-LBM [30, 29] are plotted in Fig. 6] as a function of Knudsen number. It is found that the enhancement in AGP becomes significant as the degree of rarefaction increases, while the decrement of porosity or increment of tortuosity results in the reduction of AGP. For the three levels of Sierpinski fractals and the pore body/throat system G2, LBM underestimates the AGP at most of the Knudsen numbers in the range of $[0.1,1]$ and the discrepancy between GSIS and LBM results enlarges as Knudsen number increases or the geometry becomes more complex. For the third level Sierpinski fractal L3, 

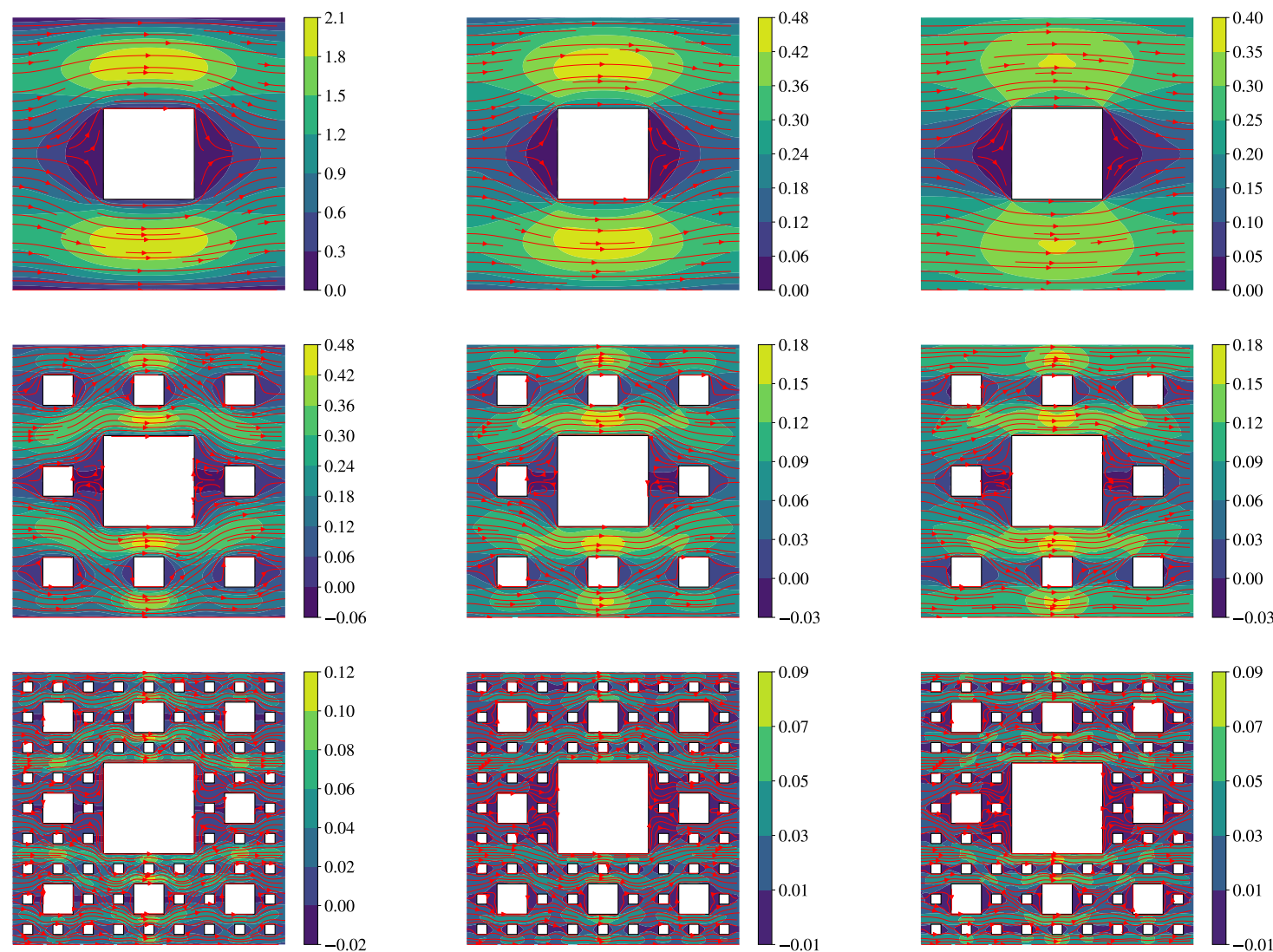

Figure 4: Horizontal velocity contours and streamlines in Sierpinski fractals. From upper to bottom, the three rows show the GSIS results obtained on the spatial grids with 1024, 4608 and 16384 triangles for the first, second and third levels, respectively. From left to right, the three columns are the results at $K n=0.01$, 0.1 and 1, respectively. The velocity space is discretized by Eq. (15) with $N_{\mathrm{v}}=48$ and $v_{\max }=4$.

the AGP from DVM is about $2.22 \times 10^{-2}$ at $K n=1$, while the one from LBM is only $4.28 \times 10^{-4}$, which is smaller than the former one by two orders of magnitude.

\subsection{Intrinsic permeability}

To quantify the Klinkenberg effect, the intrinsic permeability should be found first. To this end, we use the LBM and GSIS to calculate the intrinsic permeability at $K n=10^{-5}$. The standard LBM with the bounce-back boundary condition is used to recover the nonslip flow in the continuum regime. The inlet and outlet boundaries are treated as periodic condition as that in GSIS. More details can be found in Ref. [26]. The LBM uses $1051 \times 1051$, $1801 \times 1801$ and $2701 \times 2701$ discrete physical grids for the first, second and third level Sierpinski fractals, respectively, and $2501 \times 1001$ for the three pore body/throat geometries. The computational configurations for GSIS are given in the beginning of Sec. 4, except that here 36864 triangles are used for the Sierpinski fractal L3.

The intrinsic permeabilities $\kappa_{\infty}$ are listed in Table 2, where GSIS needs 9 to 16 iterations to obtain converged solutions. The CPU time consumed by GSIS is much less than that by LBM due to its implicit nature. For example, for the L2 geometry, GSIS takes 158 seconds 

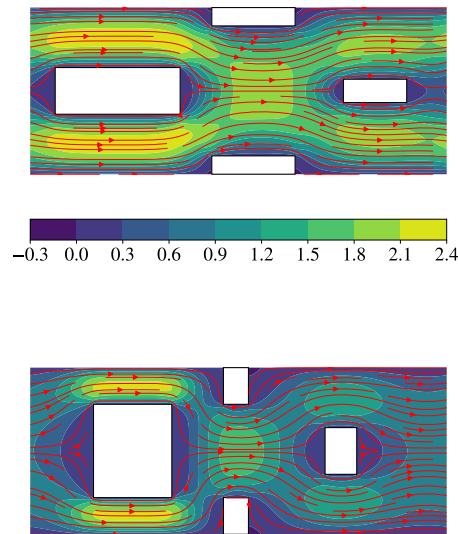

$\begin{array}{llllllllll}-0.25 & 0.00 & 0.25 & 0.50 & 0.75 & 1.00 & 1.25 & 1.50 & 1.75\end{array}$
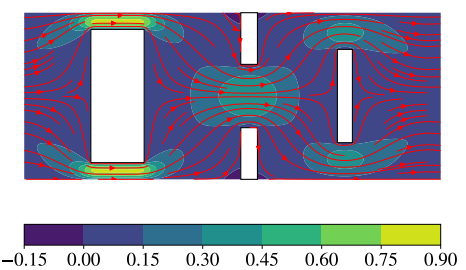
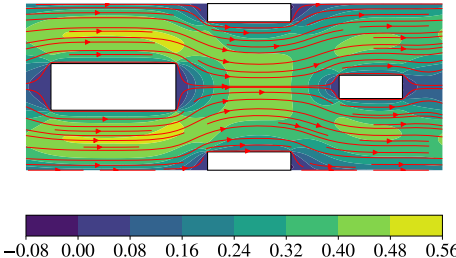

$\begin{array}{lllllllll}0.08 & 0.00 & 0.08 & 0.16 & 0.24 & 0.32 & 0.40 & 0.48 & 0.56\end{array}$

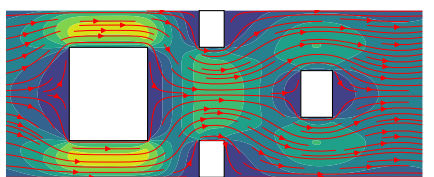

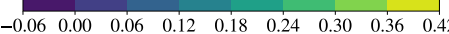

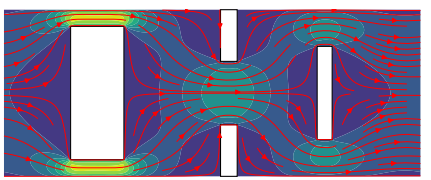

$\begin{array}{llllllllll}0.04 & 0.00 & 0.04 & 0.08 & 0.12 & 0.16 & 0.20 & 0.24 & 0.28 & 0.32\end{array}$
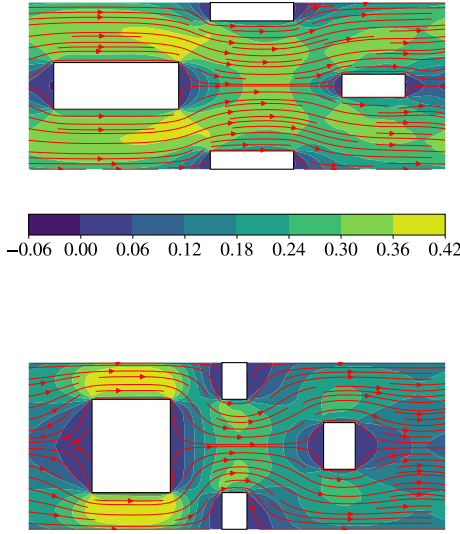

$\begin{array}{lllllllll}-0.04 & 0.00 & 0.04 & 0.08 & 0.12 & 0.16 & 0.20 & 0.24 & 0.28\end{array}$

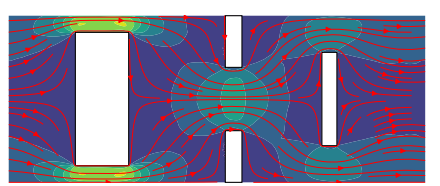

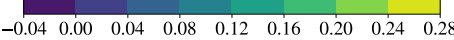

Figure 5: Horizontal velocity contours and streamlines for the pore body/throat system. From upper to bottom, the three rows show the GSIS results obtained on the spatial grids with 4088, 3488 and 3520 triangles for geometries G1, G2 and G3, respectively. From left to right, the three columns are the results at $K n=0.01,0.1$ and 1, respectively. The velocity space is discretized by Eq. 15 with $N_{\mathrm{v}}=48$ and $v_{\max }=4$.

on single processor, while LBM costs more than two days. From the table, we can see that the relative discrepancies in the intrinsic permeability between the results from GSIS and LBM are less than 1\%, except the ones for the Sierpinski fractal L3 and the pore body/throat system G3, which are about $2.2 \%$ and $2.5 \%$. This is probably due to the presence of small flow passages in these geometries, where the local Knudsen number is not so small thus the slip velocity makes the GSIS results slightly larger than those from SRT-LBM.

Our numerical results also show that, for the Sierpinski fractals, the intrinsic permeability decreases as the fractal level increases. The permeabilities for the second and third fractals are about $12.3 \%$ and $1.3 \%$ of that for the first level geometry, respectively. The reduction in $\kappa_{\infty}$ is mainly due to the reduction of porosity. For the pore body/throat geometries which

Table 2: The intrinsic permeability $\kappa_{\infty}\left(\times 10^{3}\right)$ when $K n=10^{-5}$. Sierpinski fractal model pore body/throat system

\begin{tabular}{cccccccc} 
& L1 & L2 & L3 & & G1 & G2 & G3 \\
\cline { 2 - 4 } \cline { 7 - 8 } GSIS & 8.9749 & 1.0997 & 0.1188 & & 11.1888 & 4.5427 & 0.8386 \\
LBM & 8.9149 & 1.0898 & 0.1162 & & 11.1064 & 4.4958 & 0.8176 \\
\hline
\end{tabular}



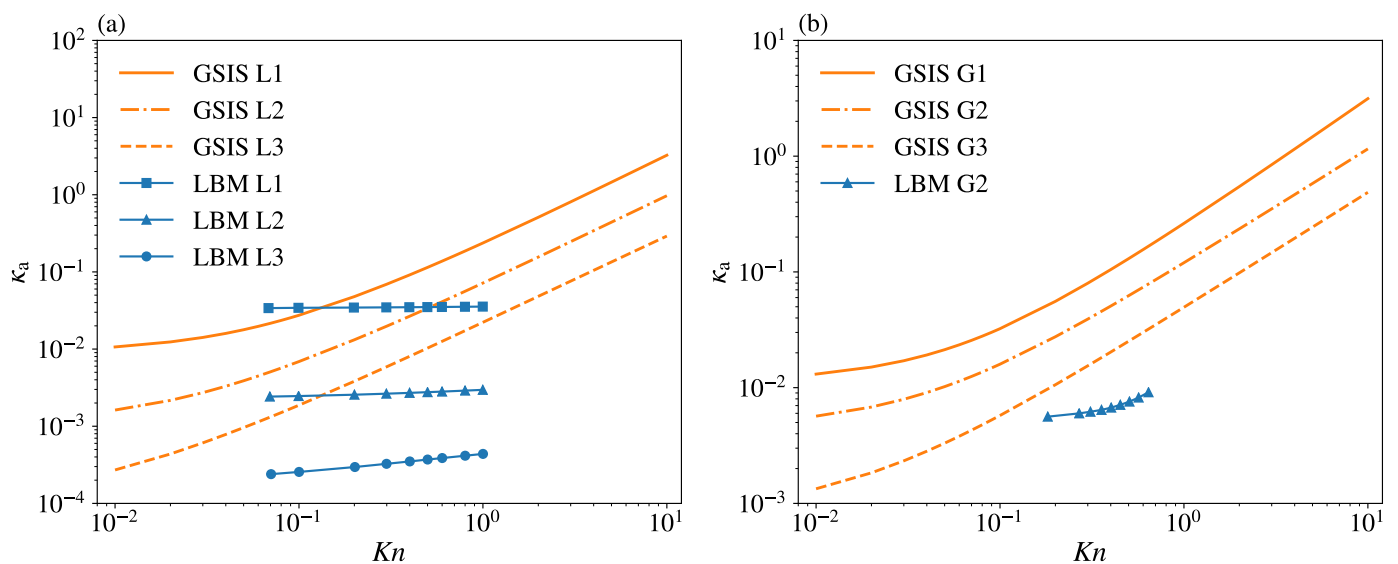

Figure 6: Comparison on the AGP obtained from the LBM and GSIS. (a) The Sierpinski fractals. (b) The pore body/throat system. LBM results in (a) and (b) are obtained from Ref. 29] and Ref. [30, respectively.
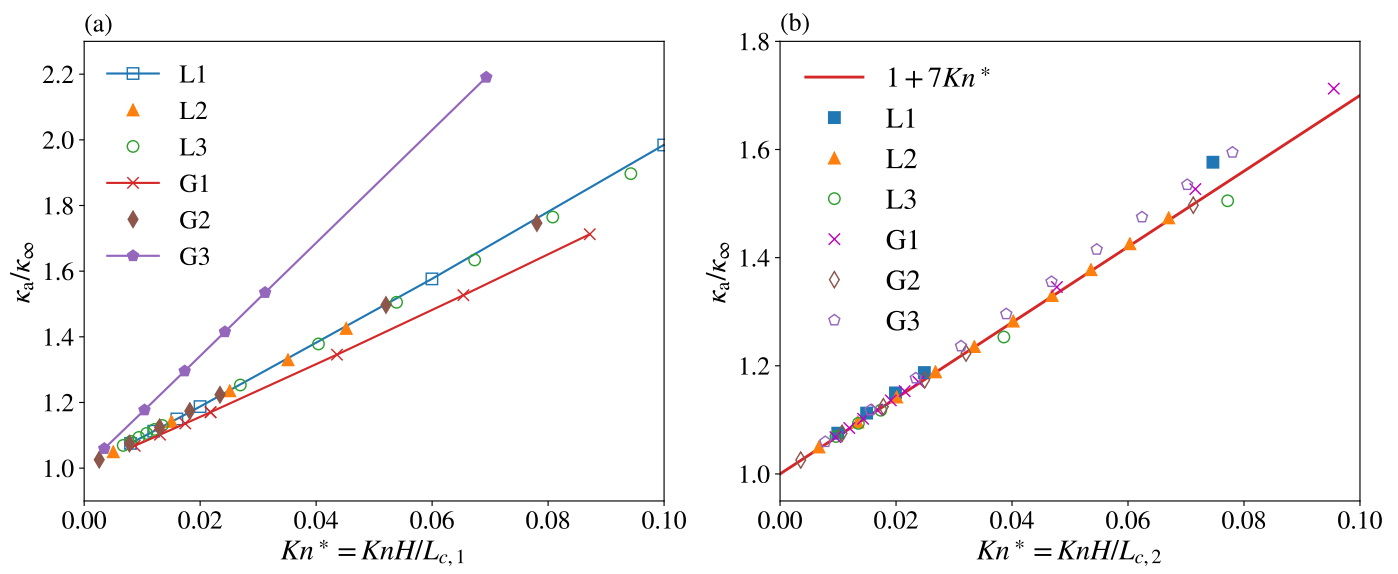

Figure 7: Variation of the ratio of the AGP to the intrinsic permeability with the equivalent Knudsen numbers $K n^{*}$. The characteristic flow length in $K n^{*}$ is defined either by (a) Eq. (28) or (b) Eq. (30).

have the same porosity, the G3 system of the largest tortuosity and narrowest flow passages possesses intrinsic permeability as small as about $7.5 \%$ and $18.5 \%$ of that for the G1 and G2 systems, respectively.

Note that the minimum height of triangle in the spatial meshes used in GSIS is larger than the mean free path of gas molecules by more than 100 times. The agreement between SRT-LBM and GSIS shows the accuracy of GSIS even with coarse physical grid. This is due to the fact that the velocity distribution function is corrected by the solution of the synthetic macroscopic equations, which asymptotically preserve the Navier-Stokes limit when $K n \rightarrow 0$.

\subsection{Slip factor in Klinkenberg correction}

Now, we quantify the ratio of the AGP to the intrinsic permeability for the six geometries in the slip flow regime. In the multi-scale flow problem, the local degree of rarefaction 
Table 3: The slope $\bar{c}$ in the linear correlation of the ratio of AGP to intrinsic permeability with the equivalent Knudsen number, $\kappa_{\mathrm{a}} / \kappa_{\infty}=1+\bar{c} K n^{*}$ for the 6 geometries shown in Fig. 1 .

\begin{tabular}{ccccccccc}
\hline$L_{c}$ defined by & L1 & L2 & L3 & & G1 & G2 & G3 \\
${$\cline { 2 - 4 }$}(28) }$ & 9.7257 & 9.4109 & 9.3039 & & 8.0684 & 9.5547 & 17.1482 \\
Eq. & 3.7373 & 7.0517 & 6.4240 & & 7.3679 & 6.9734 & 7.6100 \\
\hline
\end{tabular}

can be significantly underestimated if the characteristic flow length is chosen to be the channel height. To include the different pore structures for different porous media, Yang and Weigand [20] defined the characteristic flow length according to Eq. (3):

$$
L_{\mathrm{c}, 1}=\tau \sqrt{\frac{12 \kappa_{\infty} C}{\epsilon}} .
$$

We plot the ratio of $\kappa_{a}$ and $\kappa_{\infty}$ against the equivalent Knudsen number $K n^{*}=K n H / L_{\mathrm{c}, 1}$ based on the definition of characteristic flow length (28) in Fig. 7(a) for all the six geometries. It can be seen that the ratio of the AGP to the intrinsic permeability monotonously increases as the degree of rarefaction intensifies. The Klinkenberg correlation can be rewritten into the following form

$$
\frac{\kappa_{a}}{\kappa_{\infty}}=1+\bar{c} K n^{*},
$$

where the values of slope $\bar{c}$ obtained by linear regression are listed in Table 3. It is found that $\bar{c}$ for the three fractal geometries are close and are around 10. However, for the three pore body/throat systems, $\bar{c}$ varies significantly, which is due to the strong variation of the constriction factor $C$, see Table 1 .

We propose to estimate the characteristic flow length by the following relation without considering the constriction factor

$$
L_{\mathrm{c}, 2}=\sqrt{\frac{12 \kappa_{\infty} \tau}{\epsilon}}
$$

The ratio of the AGP to the intrinsic permeability with respect to the equivalent Knudsen number $K n^{*}=K n H / L_{\mathrm{c}, 2}$ based on the definition of characteristic flow length (30) is plotted in Fig. $7(\mathrm{~b})$. Now, the correlations between $\kappa_{a} / \kappa_{\infty}$ and $K n^{*}$ for all the geometries fall nearly in one single line. The values of slope in the linear correlation are list in Table 3. Therefore, a unique expression of the Klinkenberg correlation with the slope of about 7 is established for all the six geometries considered, when the Knudsen number characterized by the new characteristic flow length (30) is within the slip flow regime, see Figure 7(b).

\section{Conclusions}

In summary, we have investigated the rarefied gas flows through porous media based on the gas kinetic equation. First, GSIS is applied to find the steady-state solutions from the continuum to free molecular regimes, where the numerical comparisons with the LBM 
in continuum flow regime and the conventional iterative scheme in rarefied regime proved its accuracy and efficiency in the simulation of multi-scale gas flows. That is, GSIS can find converged solution within dozens of iterations at any Knudsen number, and the spatial cell size is not limited by the molecular mean free path. Second, we have shown that the MRT-LBM is not accurate in the calculation of apparent gas permeability of porous media, due to the use of limited number of discrete velocities and improper gas-surface boundary conditions. Third, based on the six porous geometries considered in this work, we have proposed a new definition of characteristic flow length, which is a function of the porosity, tortuosity and intrinsic permeability of the porous media. With this new characteristic flow length, a unique slope coefficient of about 7 in the Klinkenberg correlation has been found.

Our results could serve as benchmark data to assess the accuracy of other schemes in simulating rarefied gas flow in complex geometry over a wide range of Knudsen number. Also, the numerical method can be used to calculate the apparent gas permeability efficiently to a high fidelity.

\section{Acknowledgments}

This work is supported by the Engineering and Physical Sciences Research Council (EPSRC) in the UK under Grant No. EP/R041938/1.

\section{References}

[1] A. Islam, T. Patzek, Slip in natural gas flow through nanoporous shale reservoirs, Journal of Unconventional Oil and Gas Resources 7 (2014) $49-54$.

[2] F. Javadpour, D. Fisher, M. Unsworth, Nanoscale gas flow in shale gas sediments, Journal of Canadian Petroleum Technology 46 (10) (2007) 7.

[3] P. Guo, Dependency of tortuosity and permeability of porous media on directional distribution of pore voids, Transport in Porous Media 95 (2) (2012) 285-303.

[4] S. M. R. Niya, A. P. S. Selvadurai, A statistical correlation between permeability, porosity, tortuosity and conductance, Transport in Porous Media 121 (3) (2018) 741-752.

[5] C. F. Berg, Permeability description by characteristic length, tortuosity, constriction and porosity, Transport in Porous Media 103 (3) (2014) 381-400.

[6] F. Dullien, Porous Media: Fluid Transport and Pore Structure, second edition Edition, Academic Press, San Diego, 1992.

[7] B. Ghanbarian, A. G. Hunt, R. P. Ewing, M. Sahimi, Tortuosity in porous media: A critical review, Soil Science Society of America Journal 77 (2013) 1461-1477.

[8] L. J. Klinkenberg, The Permeability Of Porous Media To Liquids And Gases, American Petroleum Institute, New York, New York, 1941, p. 14.

[9] R. N. Moghaddam, M. Jamiolahmady, Slip flow in porous media, Fuel 173 (2016) $298-310$.

[10] W. Su, P. Wang, H. Liu, L. Wu, Accurate and efficient computation of the Boltzmann equation for Couette flow: Influence of intermolecular potentials on Knudsen layer function and viscous slip coefficient, Journal of Computational Physics 378 (2019) 573 - 590.

[11] H. Struchtrup, Macroscopic Transport Equations for Rarefied Gas Flows, first edition Edition, SpringerVerlag Berlin Heidelbrg, Berlin, 2005.

[12] J. G. Heid, J. J. McMahon, R. F. Nielsen, S. T. Yuster, Study of the Permeability of Rocks to Homogeneous Fluids, American Petroleum Institute, New York, New York, 1950, p. 17.

[13] F. O. Jones, W. W. Owens, A laboratory study of low-permeability gas sands, Journal of Petroleum Technology 32 (09) (1980) 1631-1640. 
[14] K. Sampath, C. W. Keighin, Factors affecting gas slippage in tight sandstones of cretaceous age in the Uinta basin, Journal of Petroleum Technology 34 (11) (1982) 2715-2720.

[15] L. Zhang, B. Shan, Y. Zhao, Z. Guo, Review of micro seepage mechanisms in shale gas reservoirs, International Journal of Heat and Mass Transfer 139 (2019) $144-179$.

[16] F. A. Florence, J. Rushing, K. E. Newsham, T. A. Blasingame, Improved Permeability Prediction Relations for Low Permeability Sands, Vol. SPE-107954-MS, Society of Petroleum Engineers, Denver, Colorado, U.S.A., 2007.

[17] G. E. K. Ali Beskok, Report: A model for flows in channels, pipes, and ducts at micro and nano scales, Microscale Thermophysical Engineering 3 (1) (1999) 43-77.

[18] F. Civan, Effective correlation of apparent gas permeability in tight porous media, Transport in Porous Media 82 (2) (2010) 375-384.

[19] Q. Zheng, B. Yu, Y. Duan, Q. Fang, A fractal model for gas slippage factor in porous media in the slip flow regime, Chemical Engineering Science 87 (2013) 209 - 215.

[20] G. Yang, B. Weigand, Investigation of the Klinkenberg effect in a micro/nanoporous medium by direct simulation Monte Carlo method, Physical Review Fluids 3 (2018) 044201.

[21] C. White, T. J. Scanlon, R. E. Brown, Permeability of ablative materials under rarefied gas conditions, Journal of Spacecraft and Rockets 53 (1) (2016) 134-142.

[22] Y. Kawagoe, T. Oshima, K. Tomarikawa, T. Tokumasu, T. Koido, S. Yonemura, A study on pressuredriven gas transport in porous media: from nanoscale to microscale, Microfluidics and Nanofluidics $20(12)(2016) 162$.

[23] R. Jambunathan, D. A. Levin, Advanced parallelization strategies using hybrid MPI-CUDA octree DSMC method for modeling flow through porous media, Computers \& Fluids 149 (2017) 70 - 87.

[24] L. Wu, M. T. Ho, L. Germanou, X.-J. Gu, C. Liu, K. Xu, Y. Zhang, On the apparent permeability of porous media in rarefied gas flows, Journal of Fluid Mechanics 822 (2017) 398-417.

[25] M. T. Ho, L. Zhu, L. Wu, P. Wang, Z. Guo, Z.-H. Li, Y. Zhang, A multi-level parallel solver for rarefied gas flows in porous media, Computer Physics Communications 234 (2019) $14-25$.

[26] M. T. Ho, L. Zhu, L. Wu, P. Wang, Z. Guo, J. Ma, Y. Zhang, Pore-scale simulations of rarefied gas flows in ultra-tight porous media, Fuel 249 (2019) $341-351$.

[27] L. Wu, J. Zhang, H. Liu, Y. Zhang, J. M. Reese, A fast iterative scheme for the linearized Boltzmann equation, Journal of Computational Physics 338 (2017) $431-451$.

[28] W. Su, L. Zhu, P. Wang, Y. Zhang, L. Wu, Can we find steady-state solutions to multiscale rarefied gas flows within dozens of iterations?, Journal of Computational Physics 407 (2020) 109245.

[29] H. Rostamzadeh, M. Salimi, M. Taeibi-Rahni, Permeability correlation with porosity and Knudsen number for rarefied gas flow in Sierpinski carpets, Journal of Natural Gas Science and Engineering 56 (2018) 549-567.

[30] R. Nazari Moghaddam, M. Jamiolahmady, Study of slip flow in unconventional shale rocks using lattice Boltzmann method: Effects of boundary conditions and TMAC, Transport in Porous Media 120 (1) (2017) 115-139.

[31] L. Hao, P. Cheng, Pore-scale simulations on relative permeabilities of porous media by lattice Boltzmann method, International Journal of Heat and Mass Transfer 53 (9) (2010) $1908-1913$.

[32] Z. Chai, B. Shi, Z. Guo, J. Lu, Gas flow through square arrays of circular cylinders with Klinkenberg effect: a lattice Boltzmann study, Communications in Computational Physics 8 (2010) 1052-1073.

[33] A. N. Kalarakis, V. K. Michalis, E. D. Skouras, V. N. Burganos, Mesoscopic simulation of rarefied flow in narrow channels and porous media, Transport in Porous Media 94 (1) (2012) 385-398.

[34] J. Zhao, J. Yao, A. Li, M. Zhang, L. Zhang, Y. Yang, H. Sun, Simulation of microscale gas flow in heterogeneous porous media based on the lattice Boltzmann method, Journal of Applied Physics 120 (2016) 084306.

[35] P. L. Bhatnagar, E. P. Gross, M. Krook, A model for collision processes in gases. I. Small amplitude processes in charged and neutral one-component systems, Physical Review 94 (1954) 511-525.

[36] X. He, Q. Zou, L.-S. Luo, M. Dembo, Analytic solutions of simple flows and analysis of nonslip boundary conditions for the lattice Boltzmann BGK model Journal of Statistical Physics 87 (1) (1997) 115-136. 
[37] S. Succi, Mesoscopic modeling of slip motion at fluid-solid interfaces with heterogeneous catalysis, Physical Review Letter 89 (2002) 064502.

[38] M. Sbragaglia, S. Succi, Analytical calculation of slip flow in lattice Boltzmann models with kinetic boundary conditions, Physics of Fluids 17 (9) (2005) 093602.

[39] X. W. Shan, X. F. Yuan, H. D. Chen, Kinetic theory representation of hydrodynamics: a way beyond the Navier-Stokes equation, Journal of Fluid Mechanics 550 (2006) 413-441.

[40] Y.-H. Zhang, X.-J. Gu, R. W. Barber, D. R. Emerson, Capturing Knudsen layer phenomena using a lattice Boltzmann model, Physical Review E 74 (2006) 046704.

[41] Z. Guo, C. Zheng, B. Shi, Lattice Boltzmann equation with multiple effective relaxation times for gaseous microscale flow, Physical Review E 77 (2008) 036707.

[42] W. Su, S. Lindsay, H. Liu, L. Wu, Comparative study of the discrete velocity and lattice Boltzmann methods for rarefied gas flows through irregular channels, Physics Review E 96 (2017) 023309.

[43] F. Yang, Z. Ning, H. Liu, Fractal characteristics of shales from a shale gas reservoir in the Sichuan Basin, China, Fuel 115 (2014) $378-384$.

[44] J. Zheng, W. Zhang, G. Zhang, Y. Yu, S. Wang, Effect of porous structure on rarefied gas flow in porous medium constructed by fractal geometry, Journal Natural Gas Science Engineering 34 (2016) $1446-1452$.

[45] C. Cercignani, The Boltzmann Equation and its Applications, Springer-Verlag, New York, 1988.

[46] P. Wang, M. T. Ho, L. Wu, Z. Guo, Y. Zhang, A comparative study of discrete velocity methods for low-speed rarefied gas flows, Computers \& Fluids 161 (2018) $33-46$.

[47] W. Su, L. Zhu, L. Wu, Fast convergence and asymptotic preserving of the general synthetic iterative scheme, arXiv 2003.09958 (2020) 1-22.

[48] Y. Zhu, C. Zhong, K. Xu, Implicit unified gas-kinetic scheme for steady state solutions in all flow regimes, Journal of Computational Physics 315 (2016) $16-38$.

[49] R. Sevilla, A. Huerta, Tutorial on Hybridizable Discontinuous Galerkin (HDG) for Second-Order Elliptic Problems, Springer International Publishing, Cham, 2016, pp. 105-129.

[50] W. Su, P. Wang, Y. Zhang, L. Wu, A high-order hybridizable discontinuous Galerkin method with fast convergence to steady-state solutions of the gas kinetic equation, Journal of Computational Physics 376 (2019) 973-991.

[51] W. Su, P. Wang, Y. Zhang, L. Wu, Implicit discontinuous Galerkin method for the Boltzmann equation. Journal of Scientific Computing 82 (2020) 39. 